\title{
Rapid Imaging of Unsaturated Lipids at an Isomeric Level Achieved by Controllable Oxidation
}

Jian Zhang ${ }^{\dagger, \star}$, Xinming Huo ${ }^{\dagger, \#, \star, ~ C h e n g a n ~} \mathrm{Guo}^{\dagger}$, Xiaoxiao Ma ${ }^{\dagger}$, Hanxi Huang ${ }^{\dagger}$, Jiuming He ${ }^{\S}$, Xiaohao Wang $^{\dagger}$, Fei Tang ${ }^{\dagger}, *$

$\dagger$ State Key Laboratory of Precision Measurement Technology and Instruments, Department of Precision Instrument, Tsinghua University, Zhongguancun Street, Hai Dian District, Beijing, 100084, China.

\# Tsinghua Shenzhen International Graduate School, Shenzhen University Town, Lishui Road, Xili Town, Nanshan District, Shenzhen, 518055, China.

$\S$ State Key Laboratory of Bioactive Substance and Function of Natural Medicines, Institute of Materia Medica, Chinese Academy of Medical Sciences and Peking Union Medical College, A2 Nanwei Road, Xicheng District, Beijing, 100050, China.

* Address correspondence to:

Fei Tang, tangf@mail.tsinghua.edu.cn 


\section{Contents}

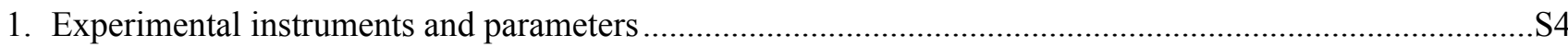

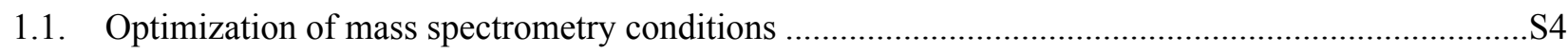

1.2. Air flow-assisted desorption electrospray ionization-mass spectrometry imaging (AFA-DESI -MSI)

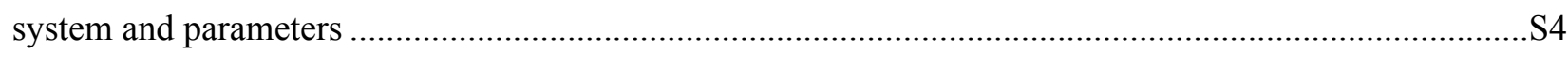

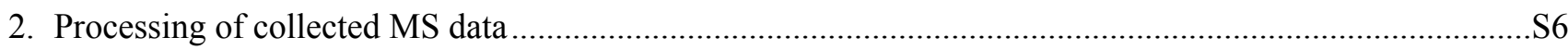

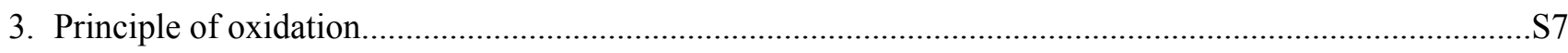

3.1. Process of alcohol generation from the oxidation of FA 18:1(9Z) ................................................ 7

3.2. Oxidation of FA 18:1(9Z) under different conditions .................................................................

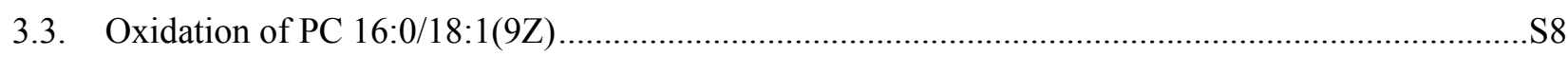

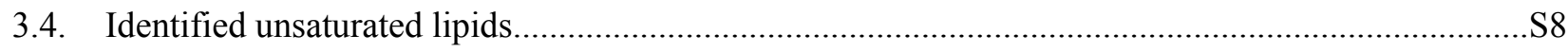

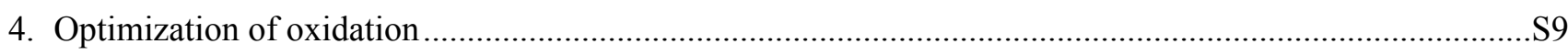

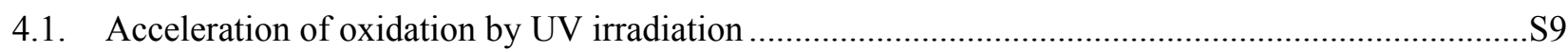

4.2. Acceleration of oxidation by low-temperature plasma jet (LTP) ....................................................S9

4.3. Full scan spectrum of FA 18:1(9Z) after $10 \mathrm{~min}$ of accelerated oxidation......................................S10

4.4. Change in the oxidation yield of FA 18:1(9Z) in air .................................................................. 10

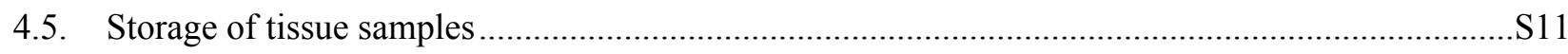

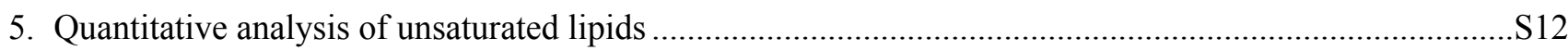

6. Oxidation-based imaging of handwriting with unsaturated lipids ............................................................

7. Oxidation-based imaging revealed a difference in the distribution of the isomers of unsaturated lipids in

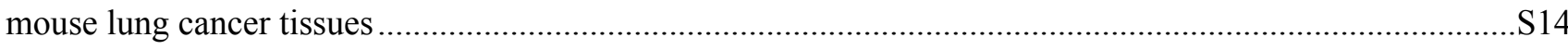

7.1. The overlapping $\mathrm{m} / \mathrm{z} 297$ signals of unfunctionalized lipids ....................................................... 14

7.2. SNRs of the characteristic ions of unsaturated lipids in nude mouse lung cancer tissues................S14

7.3. Comparison of the signal intensities of characteristic ions............................................................ 15

8. Rapid in situ spatial characterization of tumors in tissues infiltrated with cancer cells using lipid oxidationS17

8.1. SNRs of the characteristic ions of unsaturated lipids in human thyroid cancer tissues....................S17

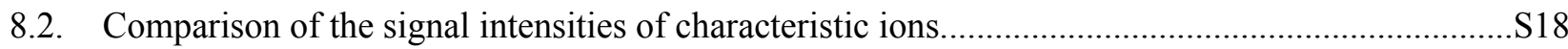




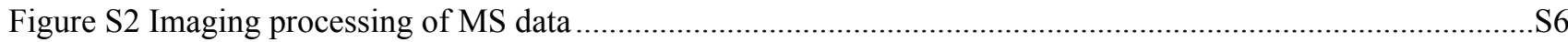

Figure S3 Detailed process of alcohol generation from FA 18:1(9Z) by oxidation ..................................................

Figure S4 The oxidation yield of FA 18:1(9Z) under different conditions...........................................................

Figure S5 CID mass spectrum of the oxidation products of PC16:0/18:1(9Z) using LTQ XL in positive ion mode S8

Figure S6 Relationship between the yield of FA 18:1 oxidation products and UV irradiation duration....................S9

Figure S7 Relationship between the yield of FA 18:1 oxidation products and LTP duration ..................................S9

Figure S8 Full scan spectrum of FA 18:1(9Z) after 10 min of oxidation at $50{ }^{\circ} \mathrm{C}$ with $60 \%$ humidity ..................S10

Figure S9 Change in the oxidation yield of FA 18:1(9Z) in the air after oxidation acceleration by moist heating..S10

Figure S10 Oxidation yield curve of fatty acids in a low-temperature environment. . $\mathrm{S} 11$

Figure S11 Quantitative calibrated curve of the relationship between ion flow signal strength and lipid amount per unit region ...... S12

Figure S12 Multi-ion monitoring chromatogram and spectrum of unsaturated lipid handwriting....... $\mathrm{S} 13$

Figure S13 The overlapping m/z 297 signals of unfunctionalized lipids in nude mouse lung cancer tissues S14

Figure S14 Scanning rows of HE stained sections of nude mouse lung cancer tissues ..$S 14$

Figure S15 Multi-ion monitoring chromatograms of unsaturated lipids in nude mouse lung cancer tissues S15

Figure S16 Comparison of the signal intensities of characteristic ions in nude mouse lung cancer tissues...... .S15

Figure S17 Scanning rows of HE stained sections of human thyroid cancer tissues..... S17

Figure S18 Multi-ion monitoring chromatograms of unsaturated lipids in human thyroid cancer tissues

Figure S19 Comparison of the signal intensities of characteristic ions in human thyroid cancer tissues. S18

Table S1 Parameters of the three-axis translation platform. S5

Table S2 The four unsaturated lipids with double bond positions identified .58 


\section{Experimental instruments and parameters}

\subsection{Optimization of mass spectrometry conditions}

Electrospray ionization (ESI) samples supplied with the instruments were used in optimization experiments to guarantee the optimisation of the mass spectrometry conditions. A QTRAP 5500 mass spectrometer was used to optimize the collision energy (CE), declustering potential (DP), entrance potential (EP), and collision cell exit potential (CXP) in the multiple reaction monitoring (MRM) mode. In the single ion monitor (SIM) mode, the LTQ XL mass spectrometer conducted automatic optimization based on the strength of $\mathrm{m} / \mathrm{z} 297.2$ and saved the result as a tune file. Then, CE was optimized in the selective reaction monitor (SRM) mode based on the strength of the total ion current, and manual fine-tuning of Act time and Act Q was carried out.

The anion mode was used, with a ESI voltage of $-4.5 \mathrm{kV}$ and a sample feeding rate of $8 \mu \mathrm{L} / \mathrm{min}$. The QTRAP 5500 mass spectrometer used atomising gas (GS1) at $35 \mathrm{~L} / \mathrm{min}$ and auxiliary gas (GS2) at $50 \mathrm{~L} / \mathrm{min}$; the LTQ Q-Exactive Orbitrap mass spectrometer used 10 arb sheath gas and 0 arb auxiliary gas. The LTQ LX mass spectrometer operated with 10 arb sheath gas and 0 arb auxiliary gas.

\subsection{Air flow-assisted desorption electrospray ionization-mass spectrometry imaging (AFA-DESI -MSI) system and parameters}

\subsubsection{AFA-DESI structural parameters}

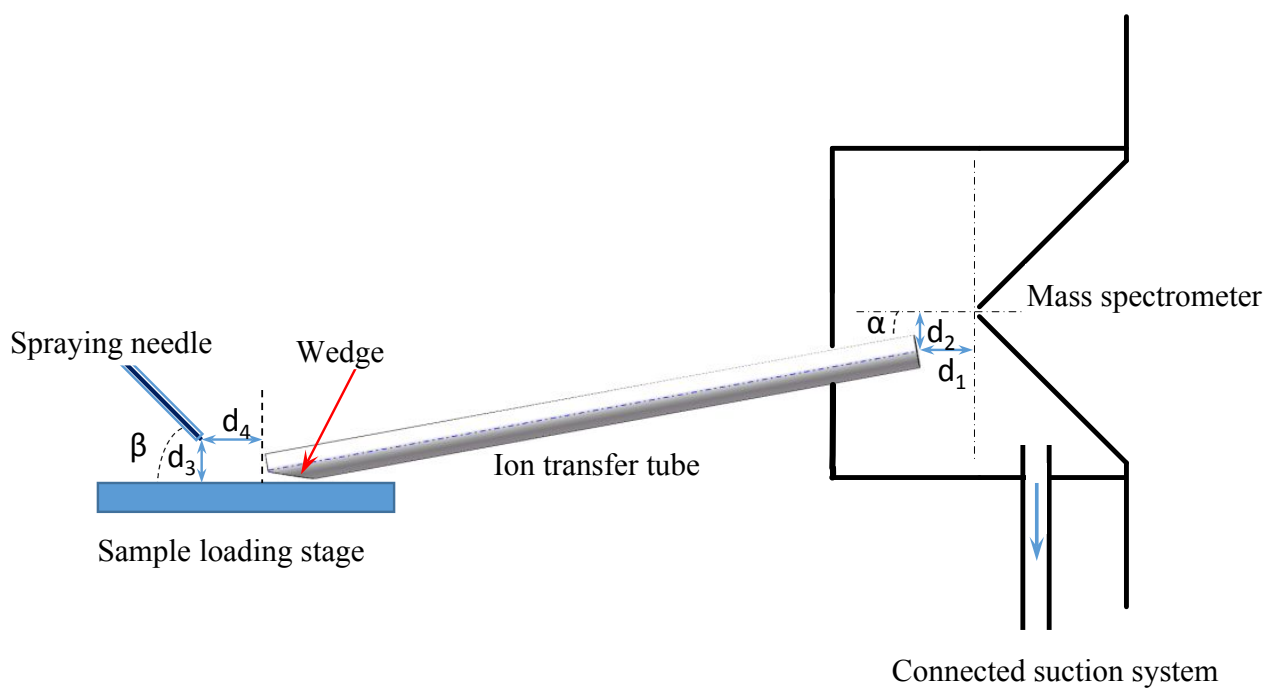

Figure S1 Structural parameters of AFA-DESI 
The AFA-DESI ion source usually consists of the DESI, an ion transfer tube, a reflux hood connected to the mass spectrometer, a sample loading stage, and a suction system. Specific parameters for debugging experiments are described below. The DESI spraying needle consisted of two layers; the inner stainless-steel capillary pipe had a $150-\mu \mathrm{m}$ outer diameter and $100-\mu \mathrm{m}$ inner diameter and the outer stainless-steel capillary pipe had a $1.6-\mathrm{mm}$ outer diameter and $300-\mu \mathrm{m}$ inner diameter. The spraying needle of the DESI was fixed onto a four-axis positioning stage (three-axis translation and one-axis rotation). The spraying needle formed a $\beta \approx 45^{\circ}$ included angle with the horizontal plane and was approximately $5 \mathrm{~mm}$ from the end of the ion transfer tube $\left(d_{4}\right)$ and approximatelyt $3 \mathrm{~mm}$ from the sample surface $\left(d_{3}\right)$. In experiments, the parameters $\left(\beta, d_{3}\right.$, and $\left.d_{4}\right)$ were adjusted slightly based on sensitivity. The ion transfer tube was a piece of stainless-steel tube, $500-\mathrm{mm}$ long with a 4-mm outer diameter and 3-mm inner diameter. At the inlet of the tube was a $15^{\circ}$ wedge notch. The transfer tube formed an $\alpha=15^{\circ}$ included angle with the axis of the taper; the end of the tube was approximately $10 \mathrm{~mm}$ (axially, $d_{1}$ ) and $3 \mathrm{~mm}$ (radially, $d_{2}$ ) from the taper. A $3-\mathrm{kV}$ DC voltage was applied to the transfer tube.

\subsubsection{Three-axis translation platform and control interface}

Table S1 Parameters of the three-axis translation platform.

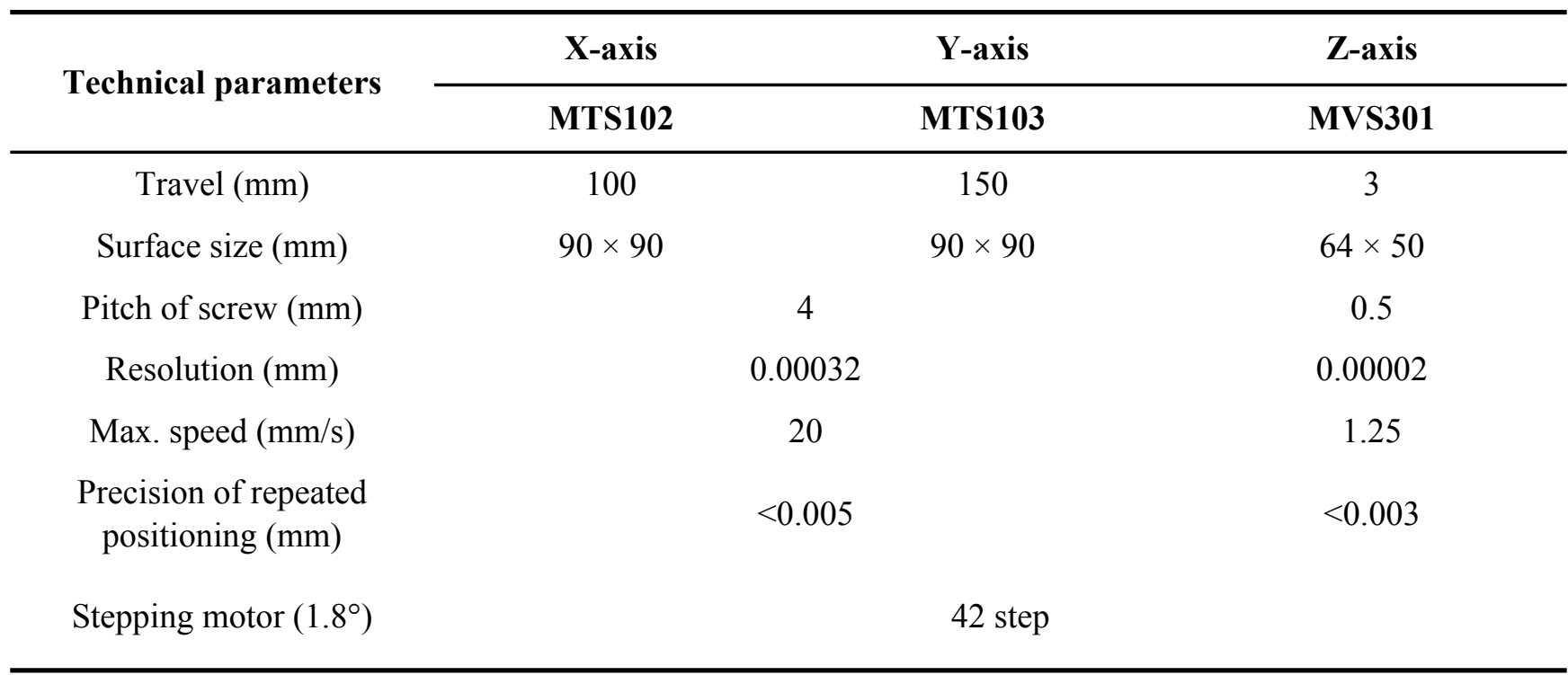




\section{Processing of collected MS data}

(a)

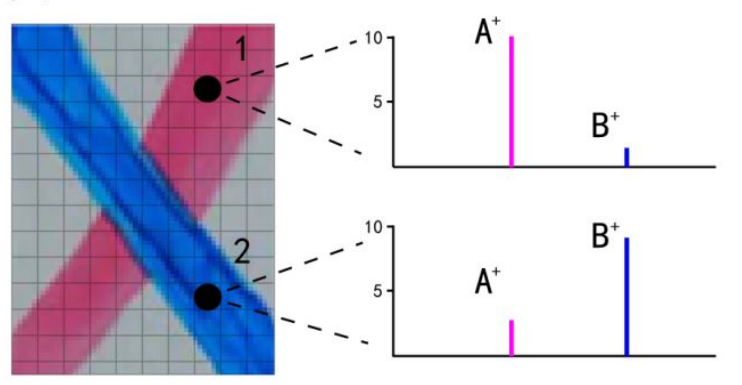

(b)

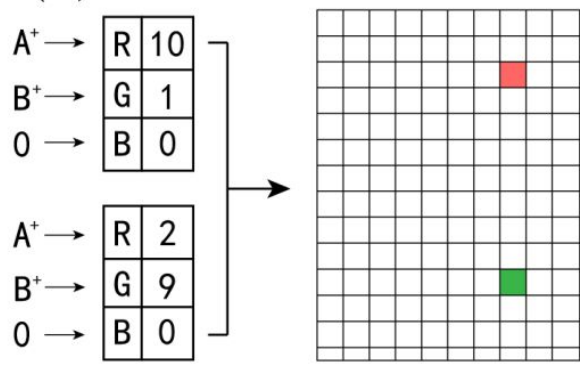

Figure S2 Imaging processing of MS data

(a) Sampling analysis of designated points by mass spectrometry; (b) signal strengths of the ions corresponding to sampling points were expressed as colors based on RGB. 


\section{Principle of oxidation}

\subsection{Process of alcohol generation from the oxidation of FA 18:1(9Z)}

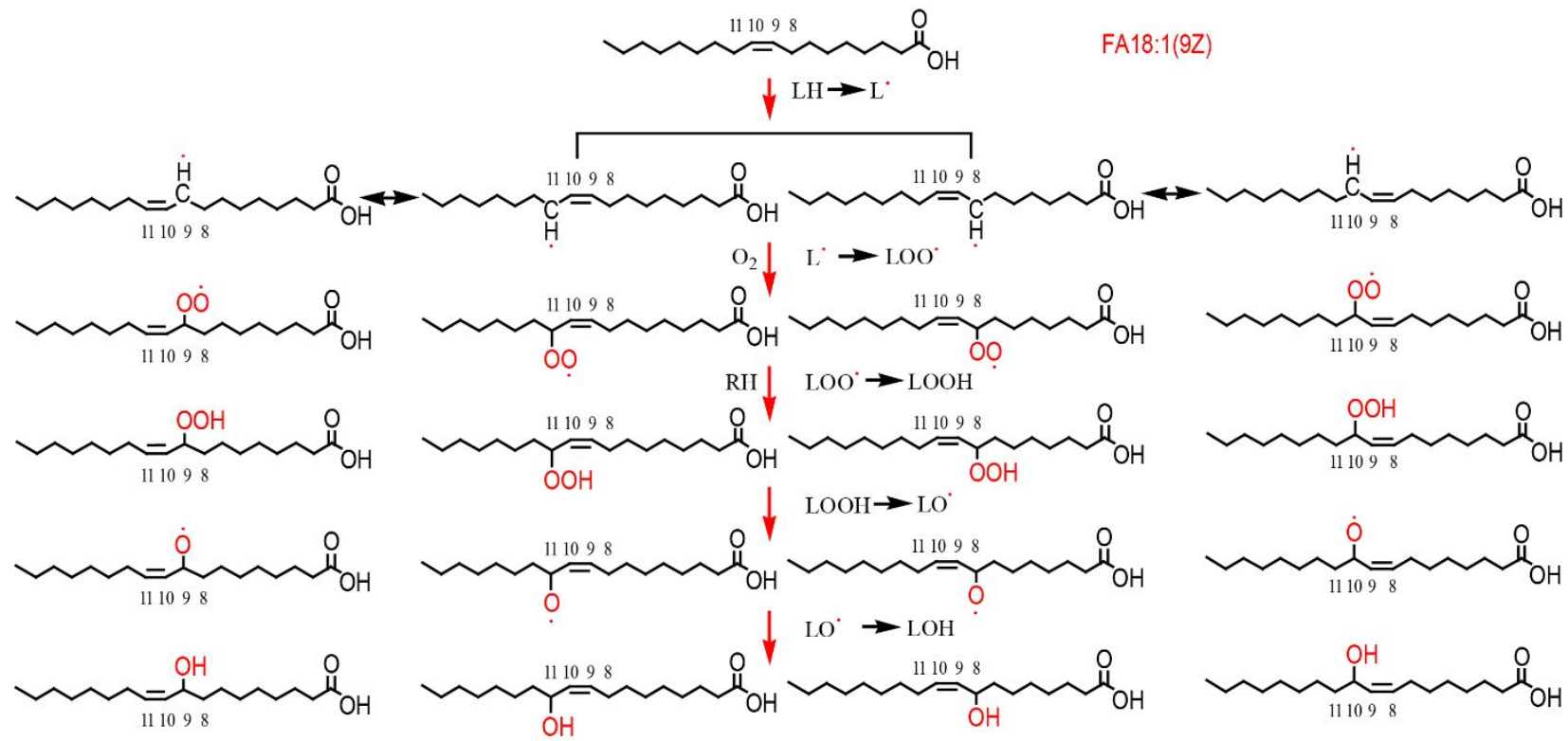

Figure S3 Detailed process of alcohol generation from FA 18:1(9Z) by oxidation

\subsection{Oxidation of FA 18:1(9Z) under different conditions}

(a)

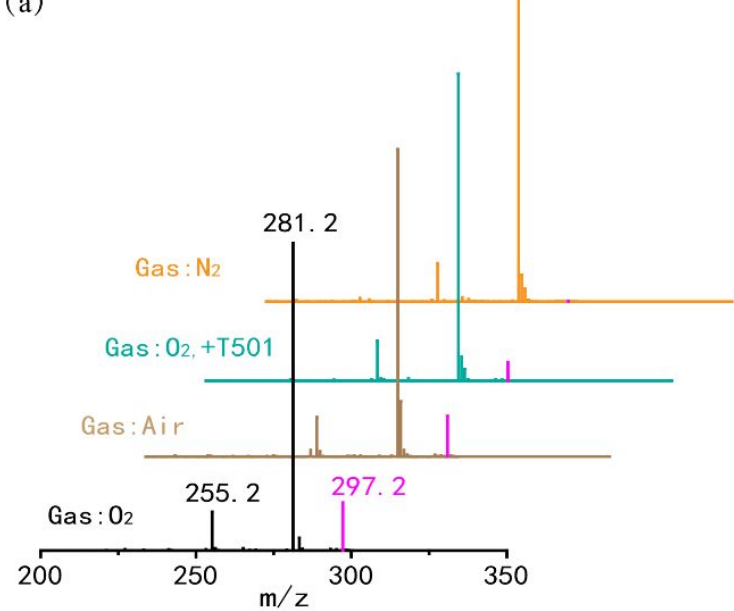

(b)

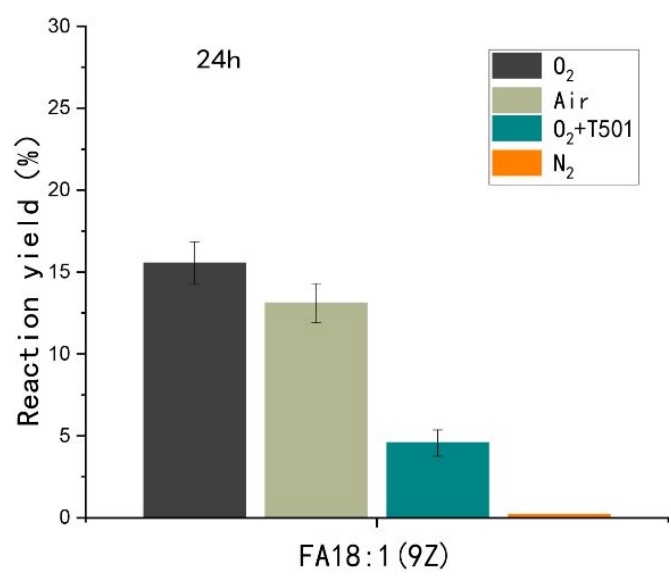

Figure S4 The oxidation yield of FA 18:1(9Z) under different conditions

(a) Negative ion mode full scan spectrum of FA18:1(9Z) after $24 \mathrm{~h}$ in a pure oxygen environment, air environment, pure oxygen environment plus oxidation inhibitor, and pure nitrogen environment. (b) Comparison of the oxidation yields of FA 18:1(9Z) under different conditions. 


\subsection{Oxidation of PC 16:0/18:1(9Z)}

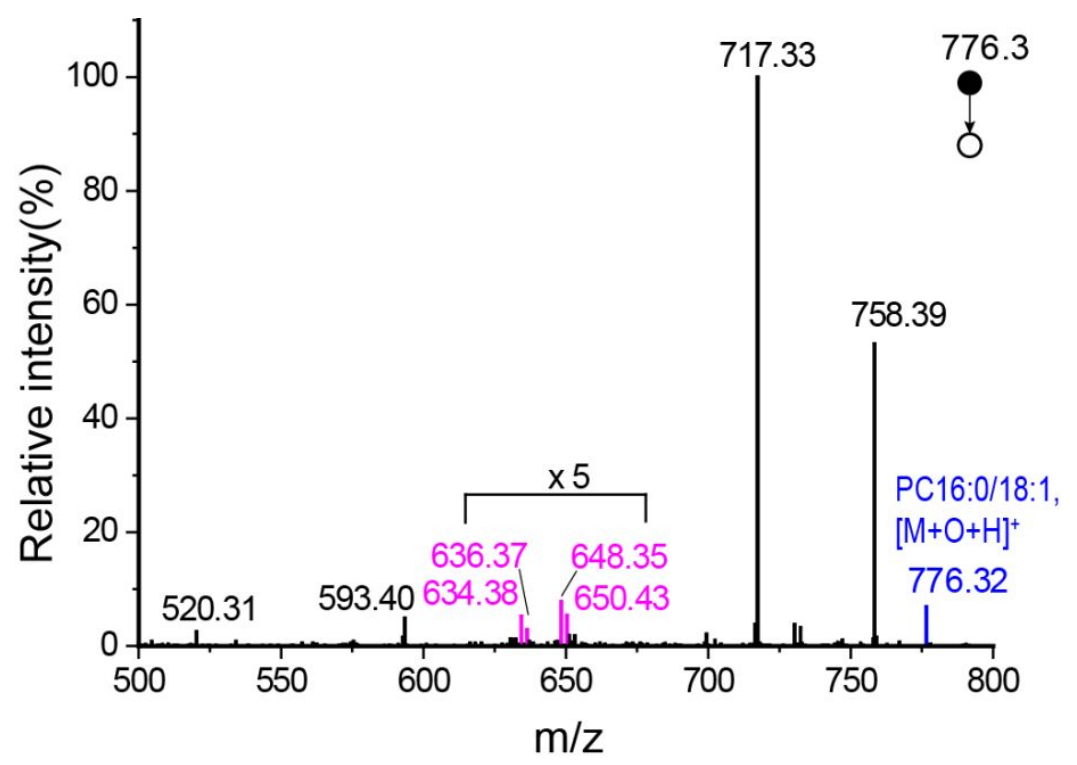

Figure S5 CID mass spectrum of the oxidation products of PC16:0/18:1(9Z) using LTQ XL in positive ion mode

\subsection{Identified unsaturated lipids}

Table S2 The four unsaturated lipids with double bond positions identified.

\begin{tabular}{cccc}
\hline $\begin{array}{c}\text { Names of } \\
\text { unsaturated lipids }\end{array}$ & Precursor ions & Oxidation products & $\begin{array}{c}\text { Characteristic ions } \\
\text { for double bond } \\
\text { position (m/z) }\end{array}$ \\
\hline FA 18:1(9Z) & 281.2 & 297.2 & $155.0,157.0,169.0,171.0$ \\
FA 18:1(11Z) & 281.2 & 297.2 & $183.1,185.1,197.1,199.1$ \\
PE 16:0/18:1(9Z) & 718.4 & 734.6 & $592.6,594.6,606.6,608.6$ \\
PC 16:0/18:1(9Z) & 760.4 & 776.3 & $634.3,636.3,648.3,650.4$ \\
\hline
\end{tabular}




\section{Optimization of oxidation}

\subsection{Acceleration of oxidation by UV irradiation}

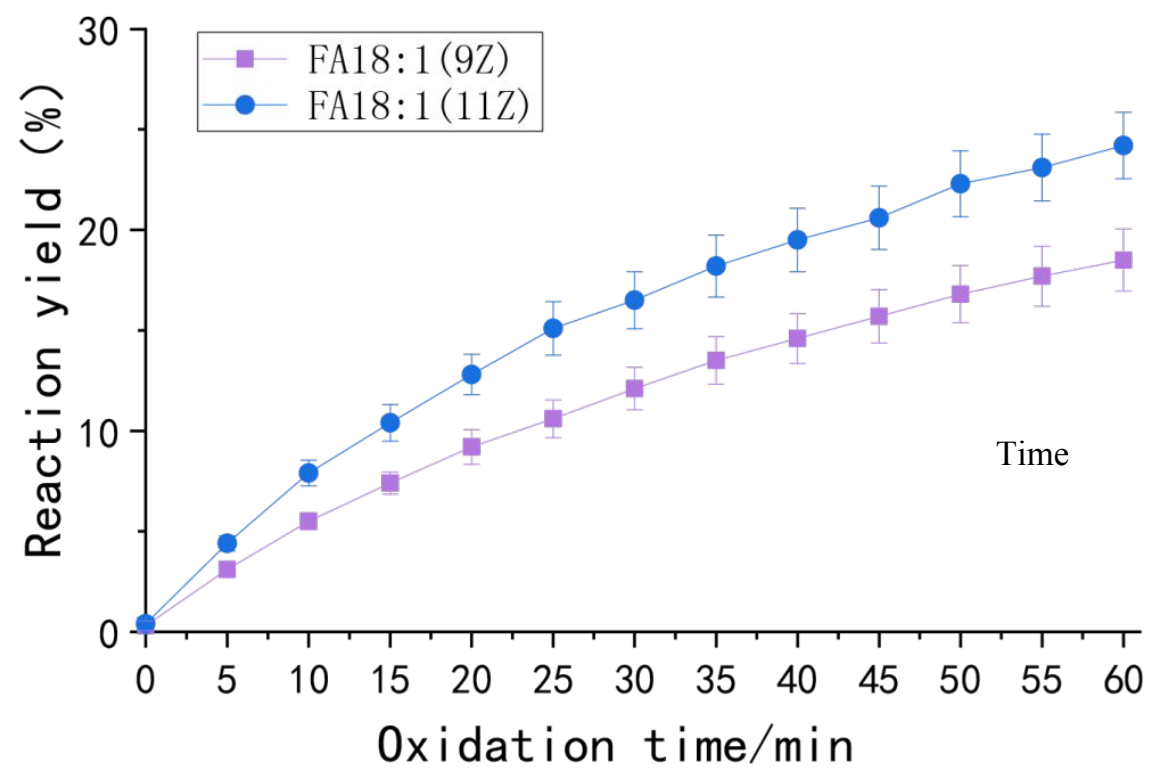

Figure S6 Relationship between the yield of FA 18:1 oxidation products and UV irradiation duration

\subsection{Acceleration of oxidation by low-temperature plasma jet (LTP)}

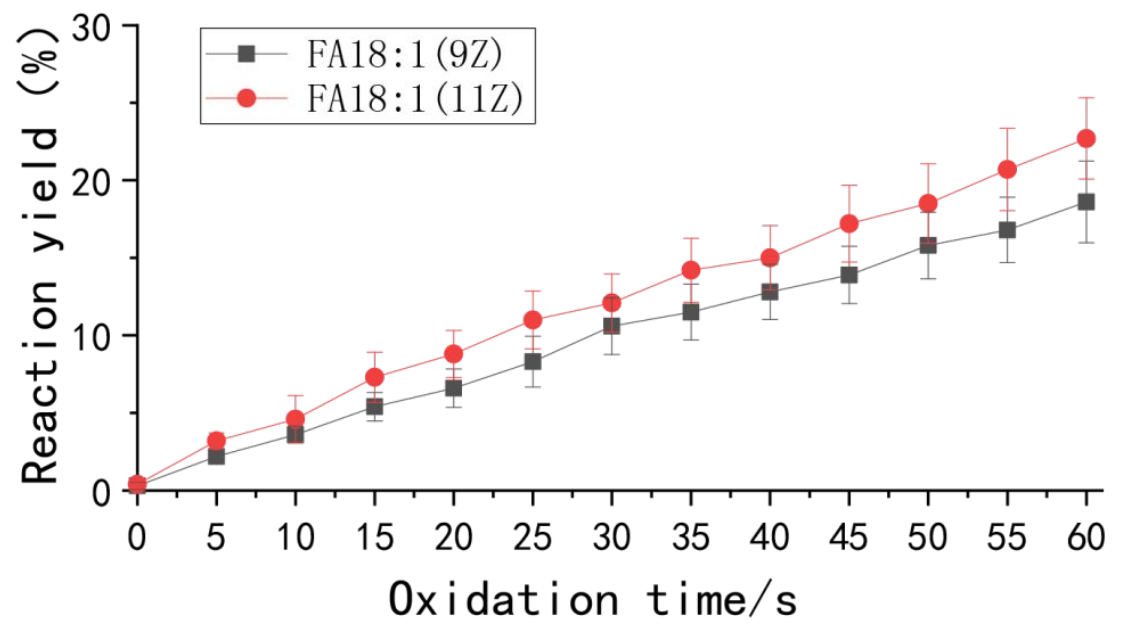

Figure S7 Relationship between the yield of FA 18:1 oxidation products and LTP duration 


\subsection{Full scan spectrum of FA 18:1(9Z) after $10 \mathrm{~min}$ of accelerated oxidation}

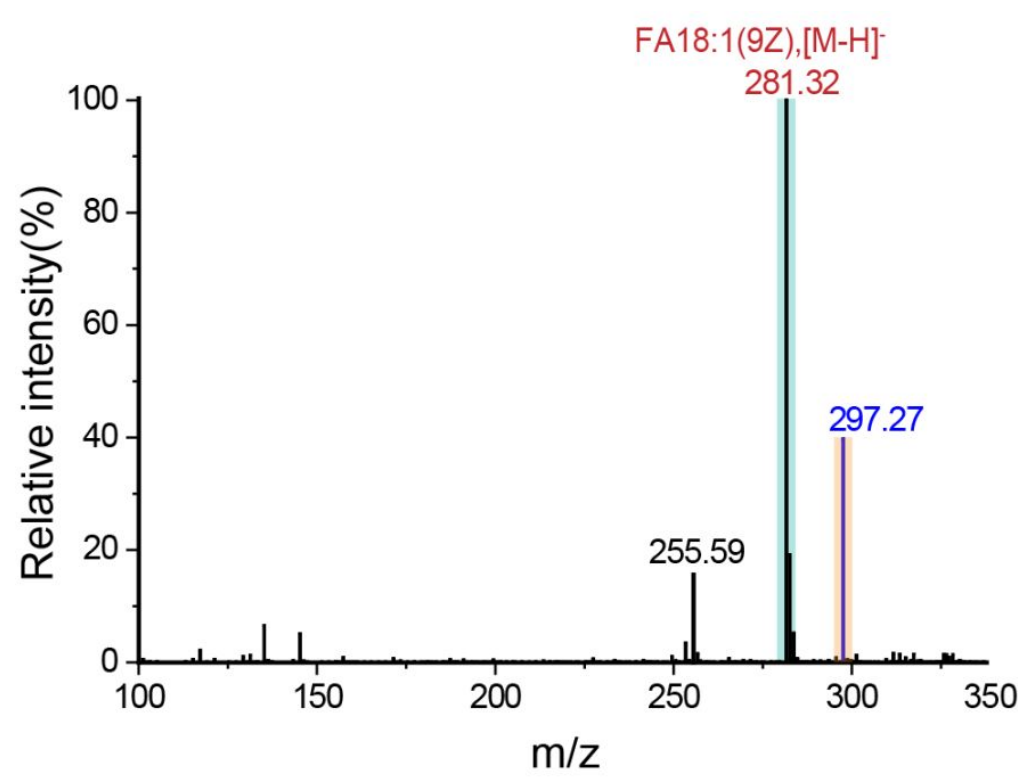

Figure S8 Full scan spectrum of FA 18:1(9Z) after 10 min of oxidation at $50{ }^{\circ} \mathrm{C}$ with $60 \%$ humidity

The ratio of $\mathrm{m} / \mathrm{z} 297$ to $\mathrm{m} / \mathrm{z} 255$ was 2.5 . Initially, $\mathrm{m} / \mathrm{z} 255$ was present at $13 \%$ of the precursor ion $m / z 281$. Therefore, the yield of $m / z 297$ was approximately $32.5 \%(2.5 \times 13 \%)$.

\subsection{Change of oxidation yield of FA 18:1(9Z) in air}

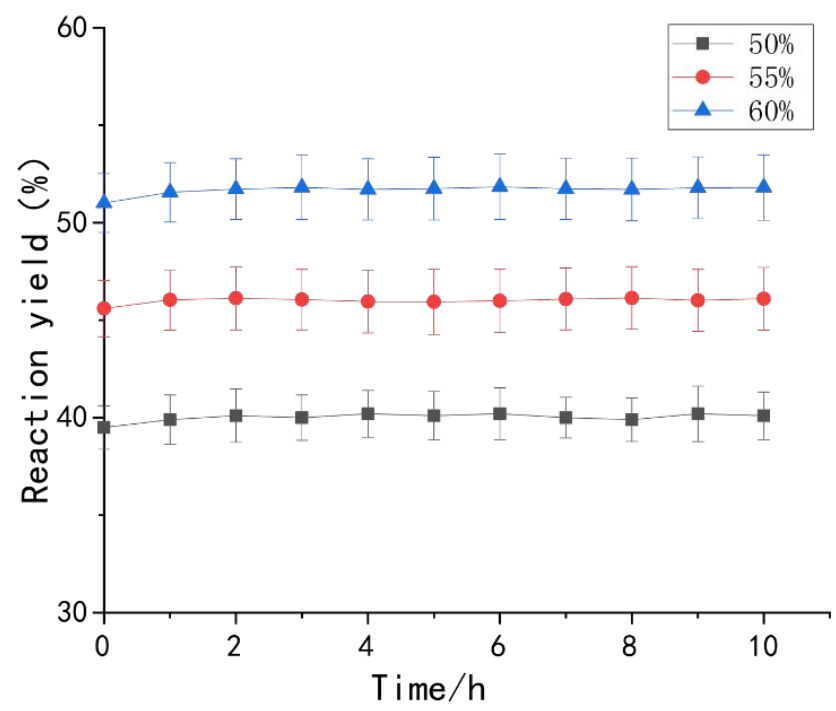

Figure S9 Change of oxidation yield of FA 18:1(9Z) in the air after oxidation acceleration by moist heating 


\subsection{Storage of tissue samples}
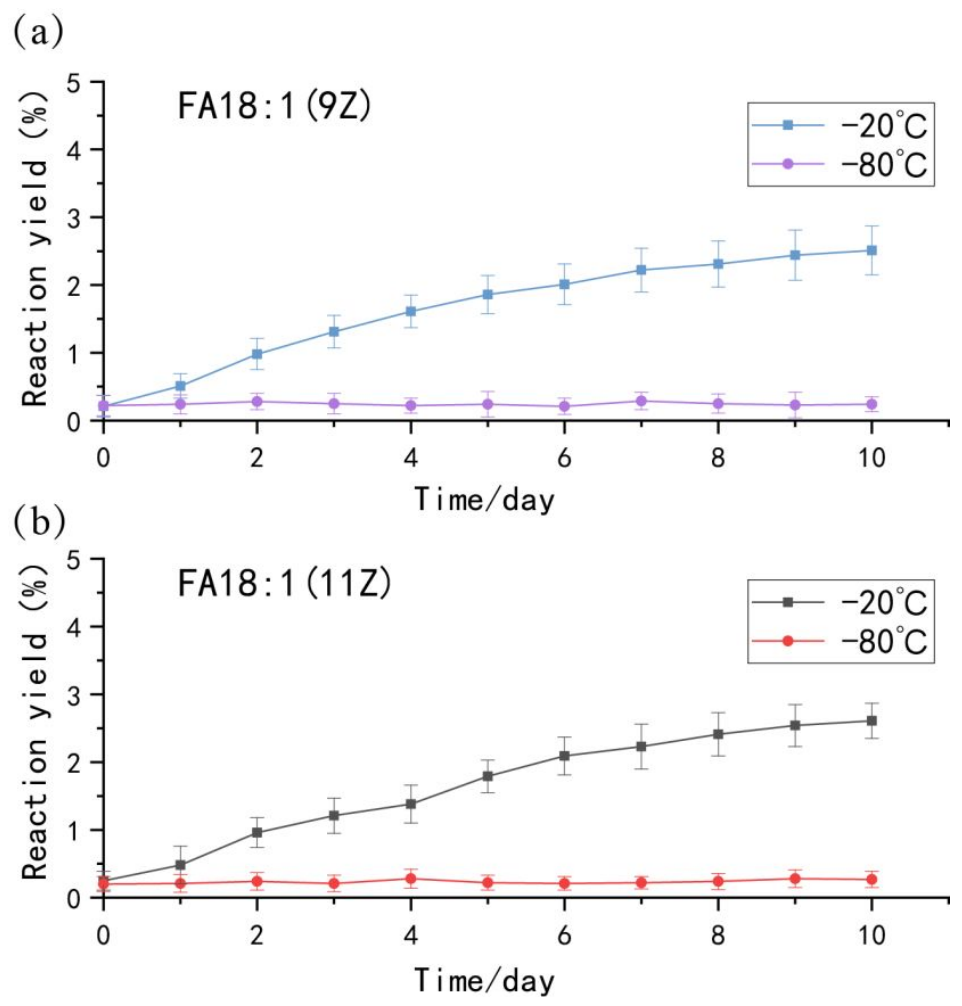

Figure S10 Oxidation yield curve of fatty acids in a low-temperature environment 


\section{Quantitative analysis of unsaturated lipids}

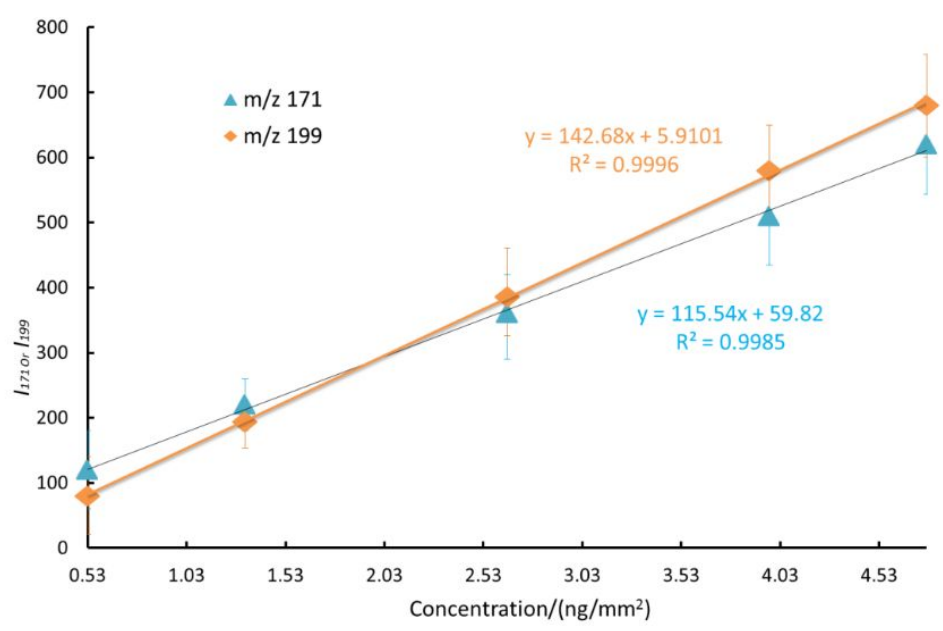

Figure S11 Quantitative calibrated curve of the relationship between ion flow signal strength and lipid amount per unit region 


\section{Oxidation-based imaging of handwriting with unsaturated lipids}

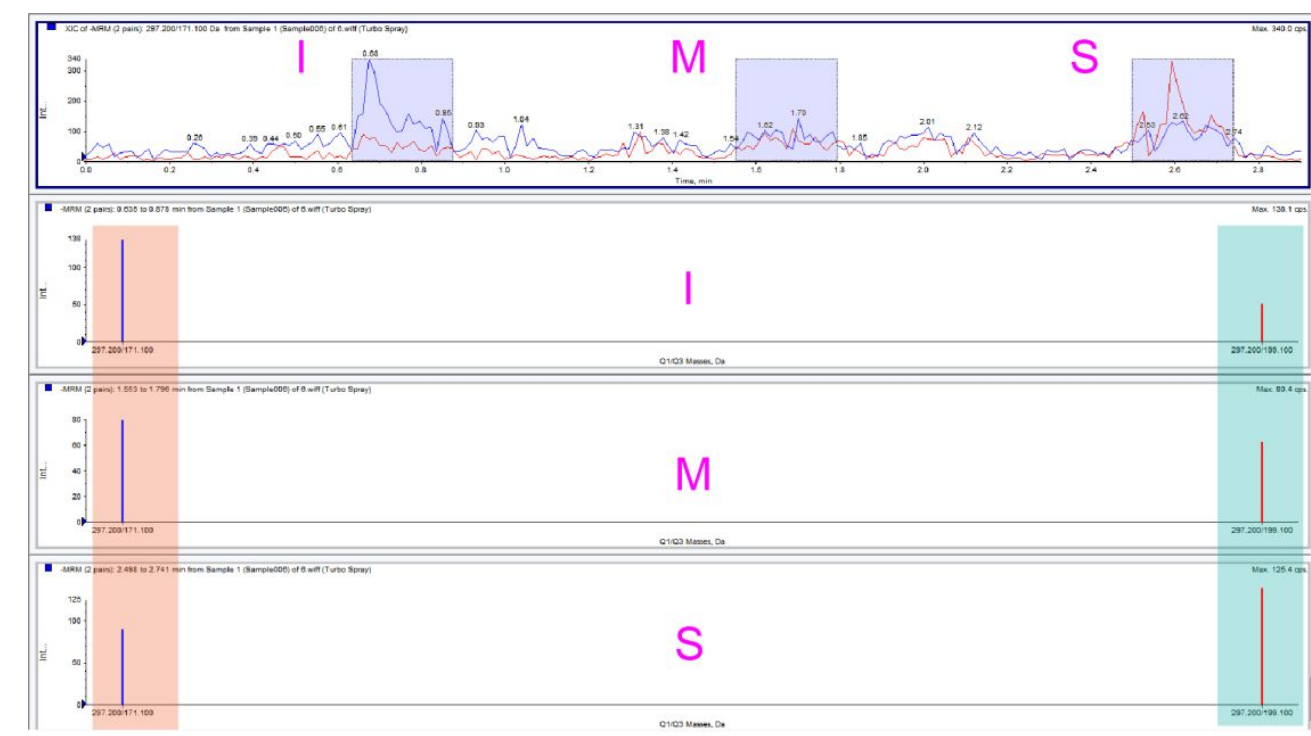

FA18:1(9Z)

FA18:1(11Z)

Figure S12 Multi-ion monitoring chromatogram and spectrum of unsaturated lipid handwriting

Oxidation imaging and scanning in the MRM mode were conducted on handwriting using unsaturated lipid isomers to obtain the ion monitoring chromatograms of two characteristic ions as shown in Figure S12. The signal-to-noise ratios (SNRs) of the characteristic ions from all three letters were generally high. When observing the different ratios of $I_{9 \mathrm{Z}}$ to $I_{11 \mathrm{Z}}$, based on cascade MS, corresponding to the letters 'I', ' $\mathrm{M}$ ' and ' $\mathrm{S}$ ', the signal strength of isomer $I_{9 \mathrm{Z}}$ decreased while that of $I_{11 \mathrm{Z}}$ increased. The ratio of the two isomers decreased from $\mathrm{I}>\mathrm{M}>\mathrm{S}$, which coincided with the imaging results. 


\section{Oxidation-based imaging revealed a difference in the distribution of the isomers of unsaturated lipids in mouse lung cancer tissues}

7.1. The overlapping $\mathrm{m} / \mathrm{z} 297$ signals of unfunctionalized lipids
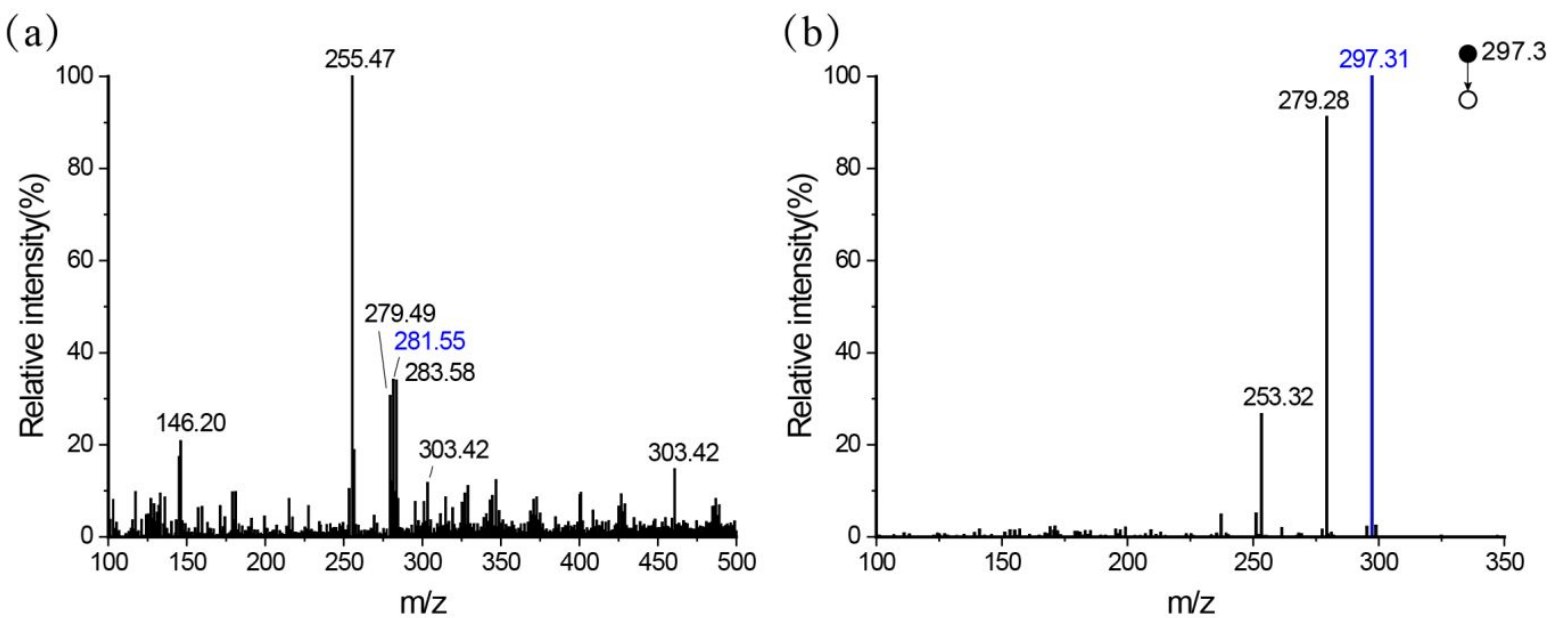

Figure S13 The overlapping m/z 297 signals of unfunctionalized lipids in nude mouse lung cancer tissues

(a) Negative ion mode full scan spectrum of lipids extracted from tissues with no oxidation using LTQ XL and (b) CID mass spectrum of the $m / z 297$ ion using LTQ XL

\subsection{SNRs of the characteristic ions of unsaturated lipids in nude mouse lung cancer tissues}

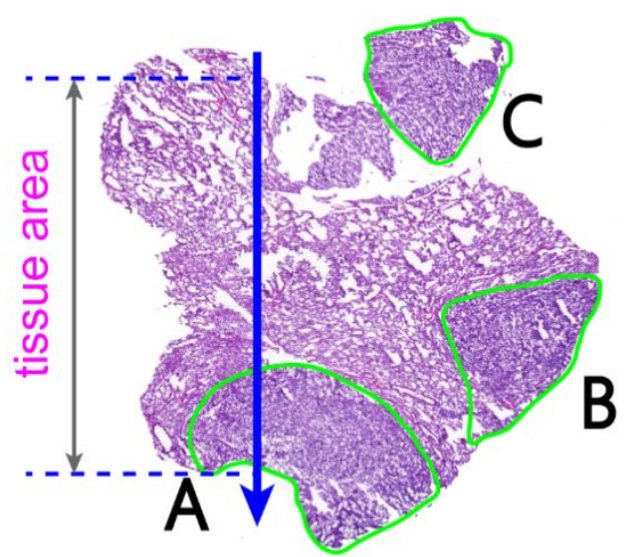

Figure S14 Scanning rows of HE stained sections of nude mouse lung cancer tissues 


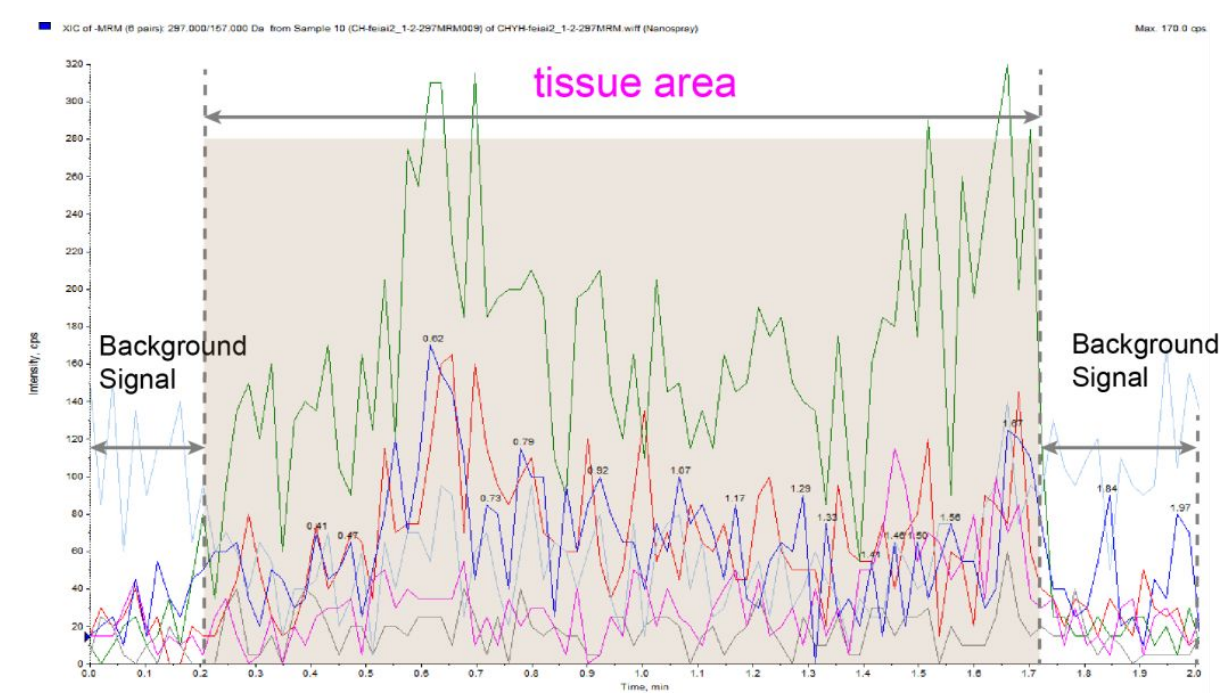

Figure S15 Multi-ion monitoring chromatograms of unsaturated lipids in nude mouse lung cancer tissues

\subsection{Comparison of the signal intensities of characteristic ions}

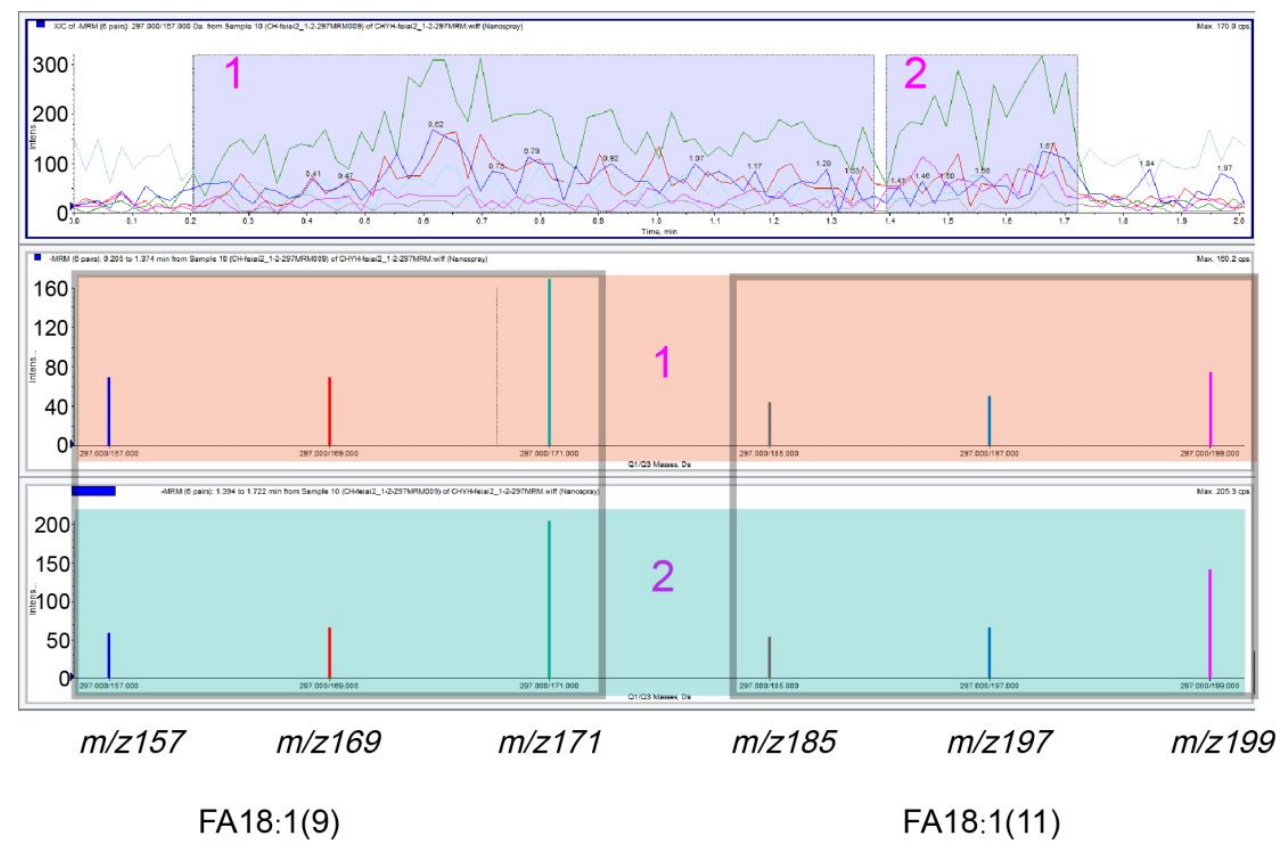

Figure S16 Comparison of the signal intensities of characteristic ions in nude mouse lung cancer tissues

The average signal strength of FA 18:1(9) consisted of the average signal strengths of $\mathrm{m} / \mathrm{z} 157$, $m / z 169$, and $m / z$ 171, while the average signal strength of FA 18:1(11) consisted of the average signal strengths of $m / z 185, m / z 197$, and $m / z$ 199. In region No. 1 the ion signal strengths of $\mathrm{m} / z 157$, $\mathrm{m} / \mathrm{z} 169$, and $\mathrm{m} / \mathrm{z} 171$ were 70,70 , and 180 , respectively, while those of $\mathrm{m} / \mathrm{z} 185, \mathrm{~m} / \mathrm{z} 197$, and $\mathrm{m} / \mathrm{z}$ 199 were 40, 50, and 70, respectively. As a result, the value of $I_{9} / I_{11}$ was approximately 2.1 in region 
No. 1. In region No. 2 the ion signal strengths of $m / z$ 157, $m / z 169$, and $m / z 171$ were 60, 70, and 200, respectively, while those of $\mathrm{m} / \mathrm{z} 185, \mathrm{~m} / \mathrm{z} 197$, and $\mathrm{m} / \mathrm{z} 199$ were 50, 70, and 110, respectively. As a result, the value of $I_{9} / I_{11}$ was approximately 1.4 in region No. 2 . 


\section{Rapid in situ spatial characterization of tumors in tissues infiltrated with cancer cells using lipid oxidation}

\subsection{SNRs of the characteristic ions of unsaturated lipids in human thyroid cancer tissues}

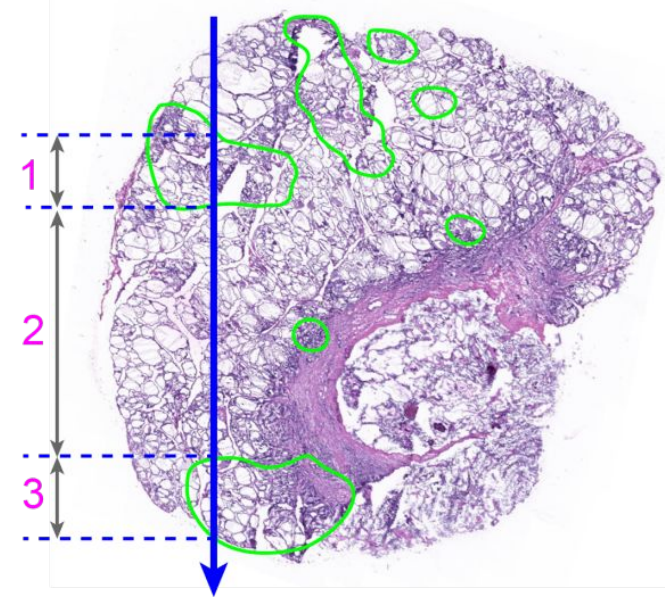

Figure S17 Scanning rows of HE stained sections of human thyroid cancer tissues

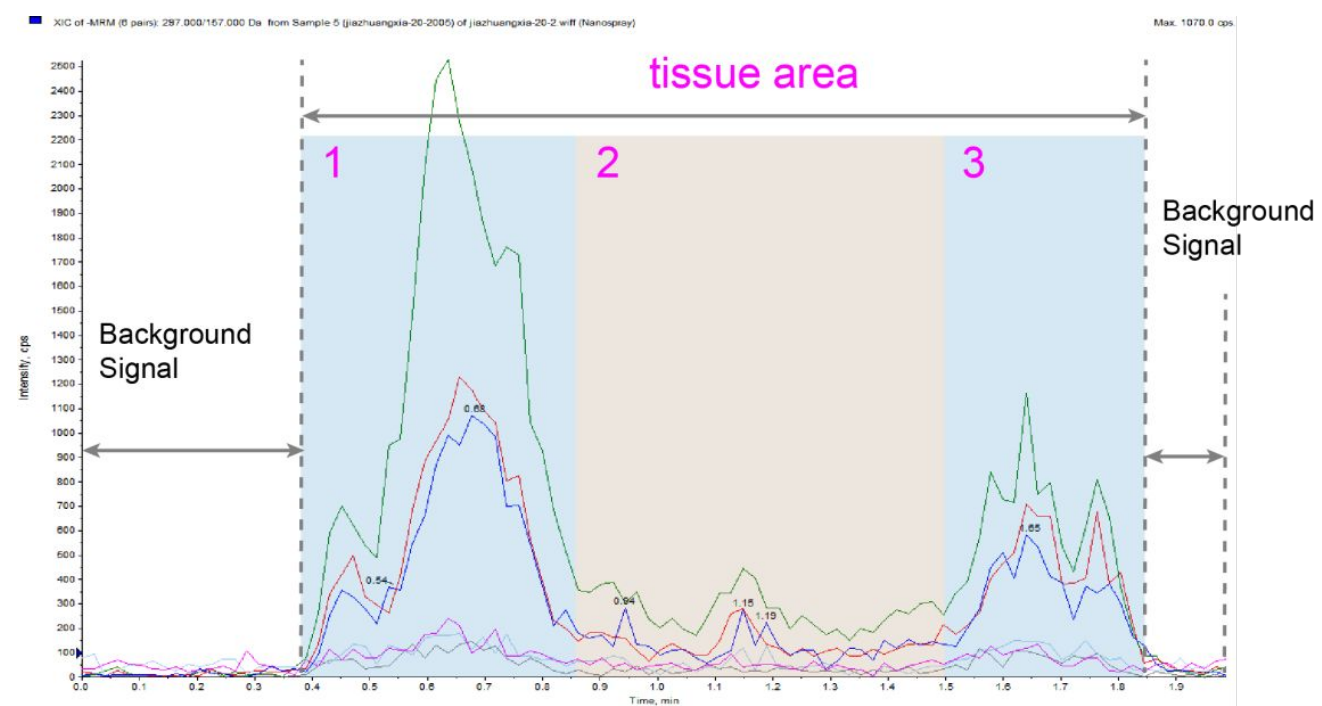

Figure S18 Multi-ion monitoring chromatograms of unsaturated lipids in human thyroid cancer tissues

Regions No. 1 and No. 3 in Figure S18 correspond to regions No. 1 and No. 3 in Figure S17, respectively, where cancer cells were concentrated; region No. 2 in Figure S18 corresponds to region No. 2 in Figure S17, where cancer cells were sparsely distributed. 


\subsection{Comparison of the signal intensities of characteristic ions.}

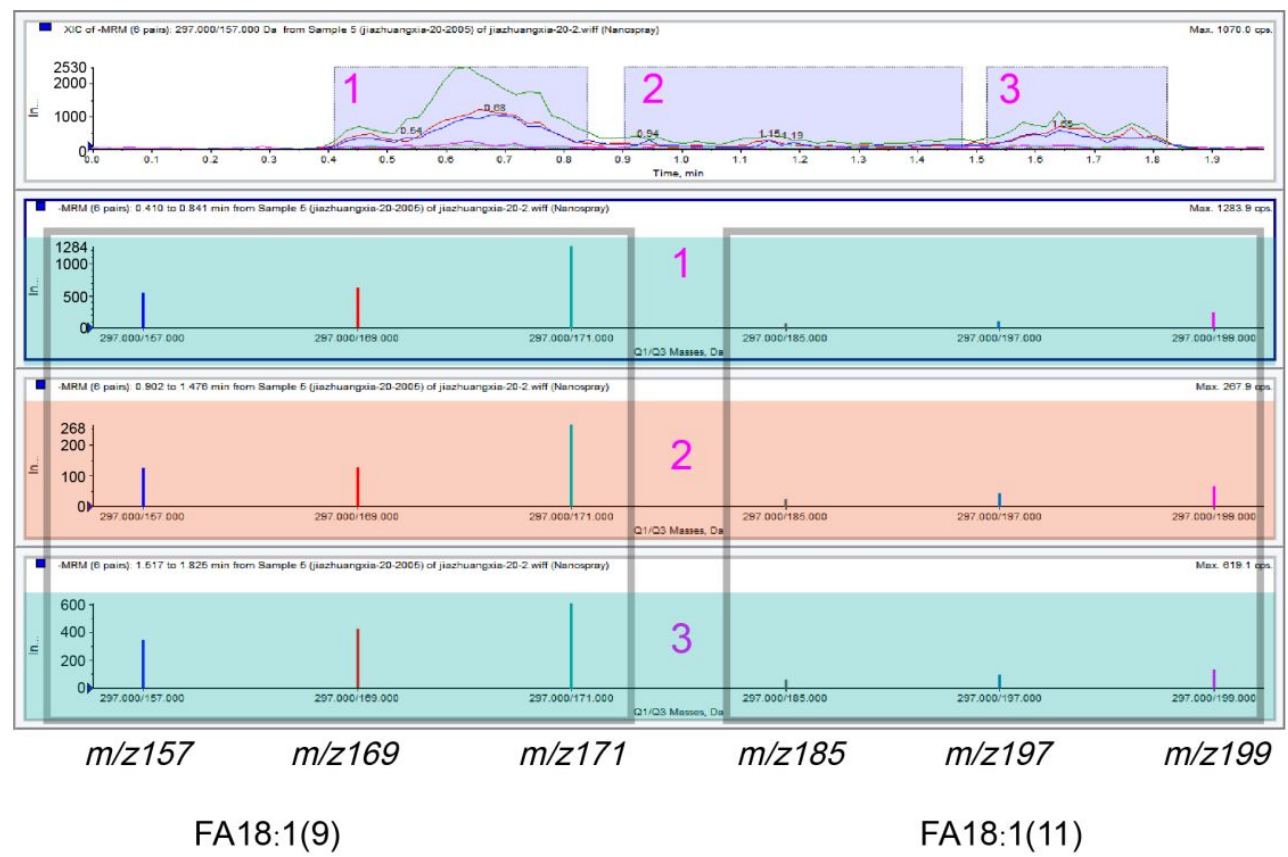

Figure S 19 Comparison of the signal intensities of characteristic ions in human thyroid cancer tissues

The average signal strength of FA 18:1(9) consists of the average signal strengths of $\mathrm{m} / \mathrm{z} 157$, $m / z 169$, and $m / z 171$, while the average signal strength of FA 18:1(11) consists of the average signal strengths of $m / z 185, m / z 197$, and $m / z$ 199. In region No. 1, the ion signal strengths of $\mathrm{m} / \mathrm{z} 157, \mathrm{~m} / \mathrm{z}$ 169 , and $\mathrm{m} / \mathrm{z} 171$ were 550,650, and 1280, respectively, while those of $\mathrm{m} / \mathrm{z} 185, \mathrm{~m} / \mathrm{z} 197$, and $\mathrm{m} / \mathrm{z}$ 199 were 100,150 , and 180, respectively. As a result, the value of $I_{9} / I_{11}$ in region No. 1 was approximately 5.7. In region No. 2, the ion signal strengths of $m / z 157, m / z 169$, and $m / z 171$ were 120, 130, and 240, respectively, while those of $\mathrm{m} / \mathrm{z} 185, \mathrm{~m} / \mathrm{z} 197$, and $\mathrm{m} / \mathrm{z} 199$ were 30, 50, and 80, respectively. As a result, the value of $I_{9} / I_{11}$ in region No. 2 was approximately 3.1. In region No. 3, the ion signal strengths of $\mathrm{m} / \mathrm{z} 157, \mathrm{~m} / \mathrm{z} 169$, and $\mathrm{m} / \mathrm{z} 171$ were 380,450 , and 650 , respectively, while those of $\mathrm{m} / \mathrm{z} 185, \mathrm{~m} / \mathrm{z} 197$, and $\mathrm{m} / \mathrm{z} 199$ were 60, 80, and 120, respectively. As a result, the value of $I_{9} / I_{11}$ in region No. 3 was approximately 5.6. The data showed that the amounts of the $C_{9}$ and $C_{11}$ isomers of both FA 18:1(9) and FA 18:1(11) were increased and the isomer ratio $\left(C_{9} / C_{11}\right)$ was higher than in the other regions. 